\title{
Casos de éxito de desarrollo rural en Europa: una primera aproximación a su aplicabilidad en España
}

Federico Soler Vayá ${ }^{1}$ Universidad Nacional de Educación a Distancia Enrique San Martín González ${ }^{2}$ Universidad Nacional de Educación a Distancia

\footnotetext{
1 fedesoler24@hotmail.com

2 esanmartin@cee.uned.es
} 


\section{Casos de éxito de desarrollo rural en Europa: una primera aproximación a su aplicabilidad en España}

Resumen. En el presente artículo se analizan cinco casos de éxito en la aplicación de políticas de desarrollo rural en distintos países de Europa con el objetivo de evaluar su posible implementación en las zonas rurales de España. Para ello, en primer lugar, se hace un repaso de los principales problemas que presentan estas zonas en el conjunto de Europa y se explica brevemente en que consiste la actual concepción de la política de desarrollo rural que se viene aplicando en la UE. A continuación, en la parte central del artículo, se detallan los cinco casos seleccionados para el estudio: las Tierras Altas de Escocia, las denominaciones de origen protegidas en Francia e Italia y las granjas ecológicas de Austria, el sistema de cooperativas agrarias en Países Bajos y Dinamarca, el turismo rural sostenible en Irlanda y Grecia y las energías renovables en los países nórdicos. Por último, se analiza la posible aplicación de estas políticas en nuestro país, teniendo en cuenta las características diferenciales y propias de cada territorio.

Palabras Claves: Desarrollo rural; Política económica; Zonas rurales.

Clasificación JEL: R11; R38; R58.

\section{Success stories of rural development in Europe: a first approach to its applicability in Spain}

Abstract. This article analyses five successful cases of the application of rural development policies in Europe with the aim of evaluating their possible implementation in the rural areas of Spain. To this end, it first reviews the main problems presented in European rural areas and briefly explains what the current conception of rural development policy in the EU consists of. After that, in the central part of the article, the five cases selected for the study are detailed: the Highlands of Scotland, the protected designations of origin in France and Italy and the organic farms of Austria, the agricultural cooperative system in the Netherlands and Denmark, sustainable rural tourism in Ireland and Greece and renewable energy in the Nordic countries. Finally, the possible application of these policies in our country is analyzed, considering the differential and specific characteristics of each territory.

Keywords: Rural development; Economic policy; Rural areas.

JEL codes: R11; R38; R58. 


\section{Introducción}

La mayor parte del mundo rural en Europa y en España sufre una serie de problemas crónicos que obligan a sus habitantes a abandonar sus pueblos de origen ante la falta de trabajo y expectativas de futuro, trasladándose a las áreas urbanas. La despoblación de amplias zonas de nuestra geografía es un despilfarro de recursos que además, tiene consecuencias económicas, sociales y ambientales negativas graves.

La política económica está obligada a proporcionar soluciones también a los problemas del mundo rural y sus habitantes. Tras muchas décadas de declive, parece claro que la identificación de las zonas rurales con el sector agrario a la hora de aplicar políticas económicas ha sido insuficiente. En consecuencia, se están popularizando nuevas políticas de desarrollo rural que apuestan por un desarrollo integral del territorio con la participación de las propias comunidades locales, en lo que se conoce como desarrollo rural endógeno o intrínseco.

El presente artículo tiene un doble objetivo: en primer lugar, presentar y difundir casos europeos de éxito que aplican estas nuevas políticas de desarrollo rural y, en segundo lugar, realizar una primera evaluación sobre la posibilidad o conveniencia de implantar (o profundizar) dichas medidas en nuestro país con el objetivo final del desarrollo rural.

El artículo, tras este primer apartado introductorio, se compone de cinco apartados. En el segundo se expone brevemente la problemática del mundo rural en Europa, explicando las diferencias existentes dentro de la UE. En el tercer apartado se resumen las principales características de las políticas de desarrollo rural modernas. Los apartados cuatro y cinco conforman el núcleo del artículo. En el apartado cuatro se analizan cinco estrategias de desarrollo rural que han tenido éxito en diferentes países europeos, mientras que en el apartado cinco se realiza un primer análisis de su aplicabilidad o conveniencia en España. Por último, en el apartado seis se resumen las principales conclusiones.

\section{La problemática del mundo rural en Europa: similitudes y rasgos específicos}

Según los datos disponibles en Eurostat ${ }^{3}$, en 2018 el 39\% de los habitantes de la UE vivía en ciudades, el $32 \%$ en zonas intermedias (suburbios o pequeñas ciudades) y el $29 \%$ lo hace en zonas rurales ${ }^{4}$. Esto supone que cerca de 150 millones de europeos viven en pequeños núcleos de población, con baja densidad demográfica y con una importante actividad agraria. Sin embargo, mientras que para 2050 se espera que la población residente en las regiones urbanas aumente en 24 millones, la población de las regiones rurales caerá en cerca de 8 millones (Eurostat, 2016). Las causas de este proceso de despoblación derivan del deterioro económico, social y medioambiental que sufre el mundo rural en el conjunto de la Unión Europea desde mediados del siglo pasado y es que, a pesar de la heterogeneidad del territorio a lo largo del viejo continente, los problemas a los que se enfrentan estas zonas, y

\footnotetext{
${ }^{3}$ https://ec.europa.eu/eurostat/web/products-eurostat-news/-/EDN-20200207-1

4 De acuerdo con la metodología actual de Eurostat sobre tipologías territoriales, estas se calculan en base a la población y la densidad poblacional existente en una cuadrícula de celdas de $1 \mathrm{~km}^{2}$. Si la población es menor de 5.000 habitantes y la densidad es inferior a 300 hab. $/ \mathrm{km}^{2}$, la celda se califica como una celda rural. Las áreas rurales son aquellas en las que más del 50\% de la población vive en celdas rurales (Eurostat, 2019).
} 
que derivan en este proceso de abandono, son generalmente los mismos en todas las regiones rurales (Frutos, 2006):

- Económicos: pérdida de competitividad de la agricultura y la ganadería tradicional por su bajo valor añadido y el aumento de las importaciones de países terceros, reducción de los ingresos de los productores por su escaso poder en la cadena de valor agroalimentaria, falta de oportunidades laborales para los jóvenes en particular y para el conjunto de los habitantes en general, aislamiento viario y de otras infraestructuras de comunicación, escasa importancia del sector secundario y del sector terciario por la falta de inversión en I+D y en nuevas tecnologías.

- Sociales: envejecimiento, desarticulación de la estructura social y de los servicios básicos, pérdida de protagonismo de los actores locales por el desplazamiento de las instituciones públicas y empresariales a los núcleos urbanos y, en consecuencia, despoblación de los municipios más pequeños.

- Medioambientales: abandono de tierras de cultivo y superficie forestal con la consiguiente degradación del medio físico, sobreexplotación de recursos hídricos, presión urbanística en determinadas zonas turísticas con el consiguiente riesgo de contaminación, vertidos y destrucción del paisaje.

Algunos autores han puesto nombre a este proceso de abandono de las zonas rurales. El profesor Esparcia (2019) acuñó el término "despoblamiento rural" para referirse al proceso de abandono de los servicios en estas zonas y la falta de oportunidades para su desarrollo económico. Por su parte, según Frutos (2006, p. 17), Rubio (1997) ya habló de los conceptos de desvitalización demográfica y desvitalización funcional para referirse al proceso de abandono de población y de actividades económicas de estas zonas derivado de la crisis agudizada en el sector agropecuario predominante en el mundo rural.

No obstante, la evolución del mundo rural en Europa no ha seguido un proceso sincrónico y el punto de partida de cada país (o región) no es el mismo (Lacambra, 2001). Puesto que, tanto la revolución agraria como la revolución industrial tuvieron lugar más tempranamente en los países nórdicos y centroeuropeos que en el sur y el este, esto provocó que el éxodo y declive rural en estas zonas se produjese mucho antes en los primeros y, en consecuencia, el nivel de industrialización y de modernización agraria es muy diferente entre países. Por ello, aunque en las regiones nórdicas existen zonas con baja densidad demográfica, su situación socioeconómica es mejor que en las regiones menos desarrolladas del sur y este de Europa. Por este motivo, la pérdida de población en las zonas rurales ha sido desigual a lo largo de los últimos años en el viejo continente, siendo mucho mayor en el sur y el este (Gráfico 1), posiblemente como consecuencia de la falta de oportunidades laborales y el mayor grado de riesgo de pobreza o exclusión social que presentan las zonas rurales de dichos países (Gráfico 2). 
Gráfico 1. Porcentaje de regiones por país que han perdido población en el periodo 1961-2011 (\% según el nivel de urbanización)

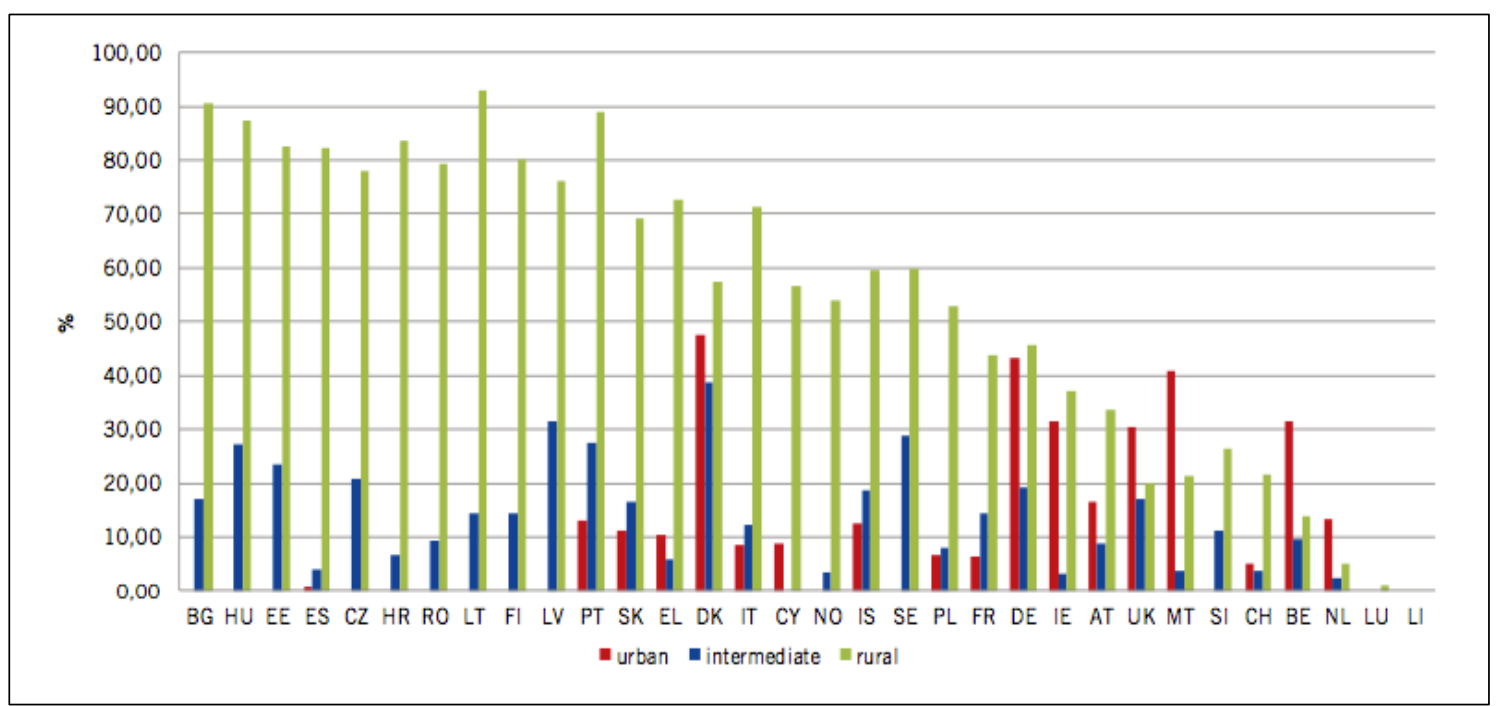

Fuente: ESPON, 2017.

Precisamente, este mayor riesgo de pobreza o exclusión social de estas zonas rurales, es otro hecho singular que muestra las diferencias entre estas dos "europas" (Figura 2). Mientras que en los países del este y del sur de Europa el riesgo de pobreza es mayor en las zonas rurales que en las urbanas o intermedias, en los países del centro y del norte esta situación es justo la contraria. Esta problemática es especialmente significativa en España en la que, a pesar de ser uno de los tres países más urbanos de la UE $(48,5 \%$ de la población vive en ciudades), tiene un elevado número de poblaciones menores de 1.000 habitantes ( $70 \%$ del total de municipios) donde el riesgo de pobreza es mucho mayor que en las zonas urbanas (31\% frente al 23\%) según Eurostat (2019b, p. 190).

Gráfico 2. Personas en riesgo de pobreza o exclusión social (2017)

(\% sobre el total de la población según nivel de urbanización)

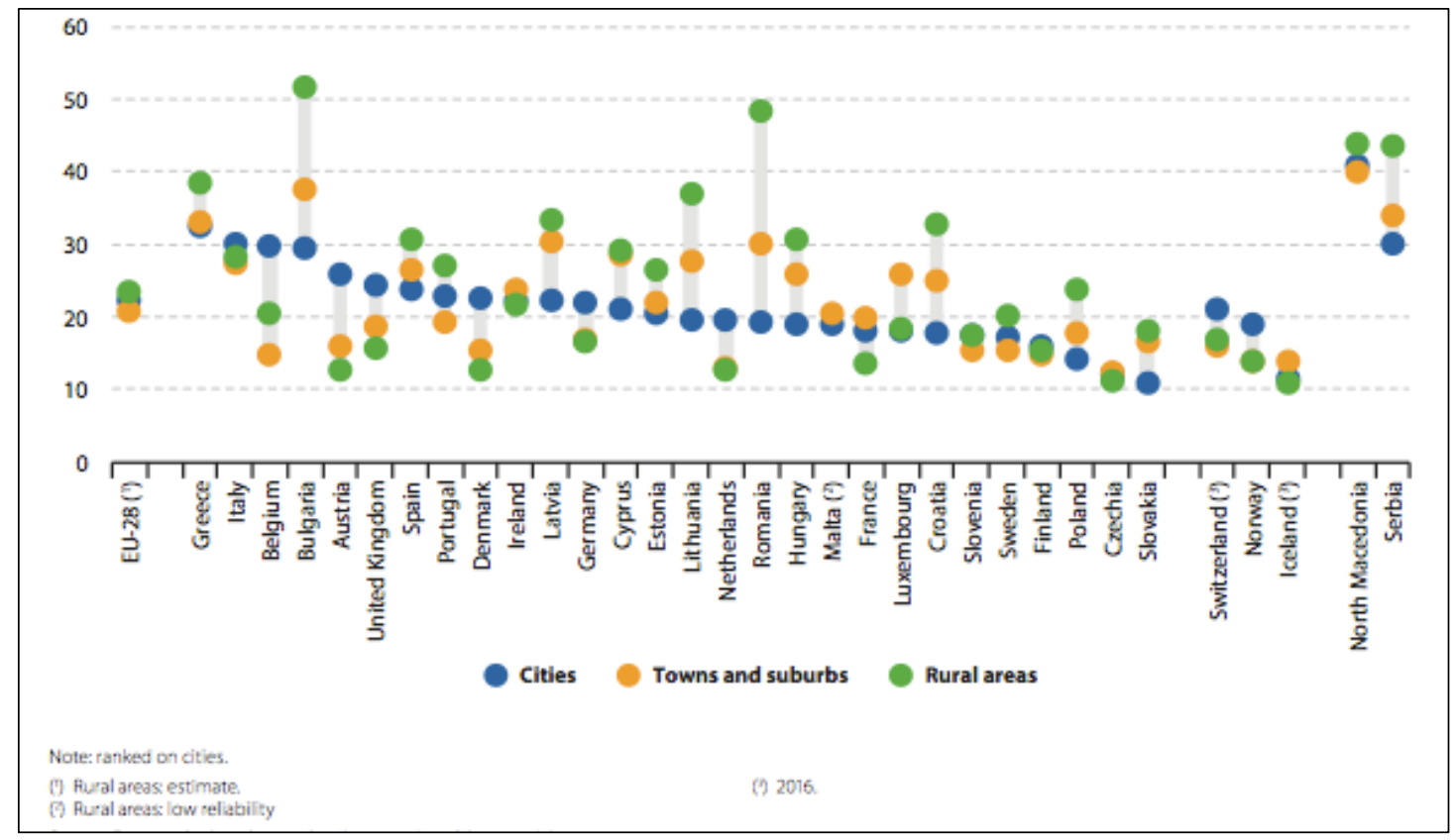

Fuente: Eurostat (2019b). 
El motivo de estas desigualdades se produce fundamentalmente por dos hechos: una tasa de empleo especialmente baja y una mayor tasa de abandono escolar temprano en estas zonas (Eurostat, 2019b, pp. 68 y 192). A pesar de estas diferencias, la tendencia durante los últimos años es similar en el conjunto de países de la UE, con un incremento del riesgo de pobreza en las zonas rurales (ESPON, 2017).

\section{La visión actual de la política de desarrollo rural}

Hasta fechas muy recientes había una coincidencia casi absoluta entre el mundo rural y el sector agrario como sujeto de análisis y la forma de actuar sobre este medio rural era, esencialmente, mediante la política agraria. Este también fue el enfoque inicial que se adoptó en Europa desde la creación de las instituciones comunitarias a través de la Política Agraria Común (PAC). Sin embargo, la creación de la PAC, y su evolución posterior no ha sido suficiente para frenar el proceso de despoblación y deterioro socioeconómico del mundo rural (Frutos, 2006). La pérdida de 4,8 millones de puestos de trabajo a tiempo completo desde el año 2000 hasta 2012 (Reig et al., 2016) es un claro ejemplo de que este proceso sigue produciéndose en el siglo XXI. En consecuencia, la visión tradicional de asimilar el mundo rural con el sector agrario ha sido abandonada progresivamente de forma paralela a la pérdida de importancia en términos de valor añadido y de empleo de este sector económico, dando lugar a una nueva concepción de las políticas públicas para la revitalización de las zonas agrarias y rurales (Rodríguez y Pérez, 2004).

El desarrollo rural implica ahora diferentes áreas y temáticas de política económica. Uno de los aspectos fundamentales de esta nueva visión para el desarrollo del mundo rural es la concepción integral del territorio y la participación de las propias comunidades locales en lo que se conoce como el desarrollo endógeno e intrínseco al territorio. Para ello la UE creó en 1988 los programas de desarrollo rural (segundo pilar de la PAC), y específicamente la metodología Leader, con la intención de implicar directamente a todas las instituciones públicas (comunitarias, nacionales, regionales y locales) mediante el desarrollo de políticas instrumentales y sectoriales bajo la estrategia "bottom-up", es decir, desde lo local hacia lo global. En este sentido, la política de desarrollo rural se ha convertido en una herramienta transversal, que aglutina a diferentes sectores, dentro de una estrategia de múltiples niveles, actores y facetas, encaminada al desarrollo socioeconómico de estas zonas (Van der Ploeg et al., 2000). El objetivo ya no es tanto el crecimiento económico sino una mejora en la calidad de vida de sus habitantes (Pezzini, 2001).

Así pues, el conjunto de países de la UE ha implementado de forma paralela esta forma de hacer política, con similitudes pero también con rasgos específicos, según la idiosincrasia de las regiones o las comunidades locales a las que han ido dirigidas. En consonancia con esta nueva concepción de la política rural, cada país, y específicamente cada región o comunidad local, siguiendo las directrices que marca la UE, ha optado por destinar sus recursos hacia unos sectores $u$ otros porque, aunque los problemas de fondo son los mismos en todos ellos, con mayor o menor intensidad, cada uno tiene unas particularidades.

Este nuevo paradigma en la manera de afrontar los problemas ha permitido desarrollar nuevas oportunidades económicas más allá de la agricultura tradicional que merecen ser objeto de estudio para tratar de extrapolar su forma de aplicación a otras regiones o comunidades. En este sentido, en el presente artículo se analizan cinco ejemplos de medidas 
y actuaciones de éxito que pueden contribuir a la elaboración de una política económica de desarrollo rural integral, intrínseca al territorio y sostenible, que permita afrontar los problemas a los que se enfrentan los habitantes de estas zonas. Aunque los casos que se analizan en el siguiente epígrafe presentan diferencias en cuanto a su ámbito de aplicación (país o región), en la forma de implementación e incluso en el sector de actividad, tienen en común que todos ellos han tenido éxito en las regiones en las que se han llevado a cabo y que han dado respuesta a alguno de los problemas fundamentales a los que se enfrentan estas zonas. Por este motivo, consideramos que es una muestra representativa que puede orientar sobre posibles cursos futuros de acción en nuestro país.

\section{Casos de éxito de estrategias de desarrollo rural en Europa}

Los casos que se analizan en este artículo son los siguientes:

- Las Tierras Altas e Islas de Escocia

- Las denominaciones de origen protegidas en Francia e Italia y las granjas ecológicas en Austria

- El sistema de cooperativas agrarias en los Países Bajos y Dinamarca

- El turismo rural sostenible en Irlanda y Grecia

- Las energías renovables en regiones rurales de Dinamarca, Suecia, Finlandia y Noruega El primer criterio que se ha utilizado para seleccionarlos era que fuesen experiencias exitosas. En segundo lugar, se han buscado iniciativas basadas en sectores y ámbitos diferentes, lo que pone de relieve el hecho de que ante problemas similares, como los tratados en los apartados anteriores, las soluciones pueden ser muy diversas. Por último, en tercer lugar, se ha procurado que hubiese diversidad geográfica dentro de Europa, puesto que la diversidad del marco natural, socioeconómico y cultural entre países siempre será mayor que la existente dentro de un mismo país y, en consecuencia, favorecerá la aparición de soluciones diferentes.

En la actualidad, hay un ejemplo en Europa que sobresale por encima del resto a la hora de afrontar los problemas del mundo rural: las Tierras Altas e Islas de Escocia. En esta región del norte de Escocia, la intervención de las administraciones públicas se ha realizado mediante la creación de una agencia de desarrollo local autónoma e independiente. Su objetivo es elaborar, desarrollar, coordinar e implementar las medidas que se deben llevar a cabo, limitando las decisiones políticas y priorizando la participación de los propios habitantes que son los que mejor conocen los problemas a los que se tienen que enfrentar (Tolón y Lastra, 2007). Los resultados no han podido ser mejores, con una recuperación y una revitalización en términos laborales y demográficos sin precedentes en una región rural en Europa.

De los casos restantes, los dos primeros tienen como objetivo incrementar la rentabilidad y el valor añadido del sector agrario mientras que los dos últimos son ejemplos de diversificación hacia actividades no agrarias de las zonas rurales.

La globalización y liberalización de los mercados agrarios aumenta la competencia y provoca una pérdida de ingresos para la agricultura y la ganadería tradicional. Ante la imposibilidad de competir vía precios con terceros países (llbery et al., 2005), normalmente menos desarrollados, los productores optan por competir vía calidad y sostenibilidad mediante denominaciones de origen e indicaciones geográficas protegidas (DOP o IGP) o con la agricultura y la ganadería ecológica. Las DOP e IGP han sido utilizadas con éxito en Francia e Italia mientras que en Austria se han promovido granjas ecológicas. Otra consecuencia del 
proceso de globalización y liberalización es el aumento de la longitud de las cadenas de distribución y la pérdida de poder negociador de los productores agrarios locales frente al resto de eslabones de la cadena de distribución. Una buena alternativa para mantener o incrementar la relevancia de los productores agrarios locales en el sistema comercial es el cooperativismo. En los Países Bajos y Dinamarca el buen funcionamiento de estas organizaciones de economía social ha permitido generar economías de escala en la producción agrícola así como aumentar su poder de negociación y comercialización en los mercados locales, nacionales e internacionales (Bijman et al., 2012).

Sin embargo, para conseguir frenar y superar los principales problemas del mundo rural es posible que no sea suficiente con actuar sobre el sector primario. Rico (2005), por ejemplo, aboga por explotar otras alternativas económicas en los sectores secundario y terciario. Una de las principales opciones es el turismo rural (Plaza y Araque, 2000; Jiménez, 2000). Aunque en España son muchos los ejemplos de proyectos destinados al desarrollo de esta actividad económica, los casos aquí mostrados ponen el énfasis en la sostenibilidad de este tipo de turismo mediante la valorización de los productos y la gastronomía local como factor de atracción en Irlanda y Grecia. Por último, otro sector hacia el que las regiones rurales pueden diversificarse, y en el que tienen ventajas competitivas frente a las zonas urbanas, es el desarrollo de energías renovables. El ejemplo aquí estudiado muestra como los habitantes de las zonas rurales escandinavas no solo se han dedicado a la generación de energía renovable sino que, además, se han convertido en emprendedores, aumentando su participación en la cadena de valor de los productos energéticos mediante la propiedad de recursos, su distribución y su comercialización directamente en el mercado, rompiendo el aislamiento del mundo rural respecto a las zonas urbanas y la visión de subsidiariedad (Bahadillo, 2005; De Pablo y Mirta, 2002).

A continuación, explicaremos con un mayor grado de detalle los casos seleccionados.

\subsection{Las Tierras Altas e Islas de Escocia}

Las Tierras Altas e Islas (Highlands and Islands ${ }^{5}$ ) es una región del norte de Escocia (ver Figura 1) que sufrió un fuerte proceso de despoblación y declive económico desde el siglo XVIII hasta mediados del siglo XX como consecuencia de su mala comunicación con los principales núcleos de población, su orografía y su atraso en el proceso de industrialización del país.

Tras perder más del $20 \%$ de la población desde mediados del siglo XIX hasta mediados del siglo XX, en 1965 el gobierno escocés aprobó la Ley de desarrollo para las Tierras Altas e Islas de Escocia (Highlands and Islands Development Scotland Act) con la que se creó una agencia pública de desarrollo (Highlands and Islands Development Board ${ }^{6}$ ) con autonomía propia respecto al resto de administraciones públicas. El objetivo no era otro que conseguir revertir la situación de atraso económico y social de esta región mediante un planteamiento holístico del territorio, evitando enfoques sectoriales y sesgados políticamente e implicando a los propios habitantes de esta zona en la toma de decisiones estratégicas para su desarrollo.

Para ello, se dotó a la agencia de financiación y profesionales de diferentes ámbitos con arraigo al territorio mediante convocatorias públicas de empleo (aunque sus trabajadores no son funcionarios) y se establecieron acuerdos de colaboración con otras entidades

\footnotetext{
${ }^{5}$ La información de este apartado ha sido obtenida del informe SSPA (2017).

${ }^{6}$ Esta agencia fue el precedente de la actual, la Highlands and Islands Enterprise creada a principios de los años 90.
} 
públicas, empresas, universidades, administraciones locales, regionales y europeas. Todo ello bajo una concepción integradora del territorio, un horizonte temporal a medio y largo plazo y un contacto directo con la realidad local (personas y empresas) para buscar la generación de oportunidades de crecimiento endógeno y sostenible. Además, al ser un organismo público había de someterse a procedimientos de control para evaluar el adecuado uso de los fondos públicos.

Figura 1. Tierras Altas e Islas de Escocia

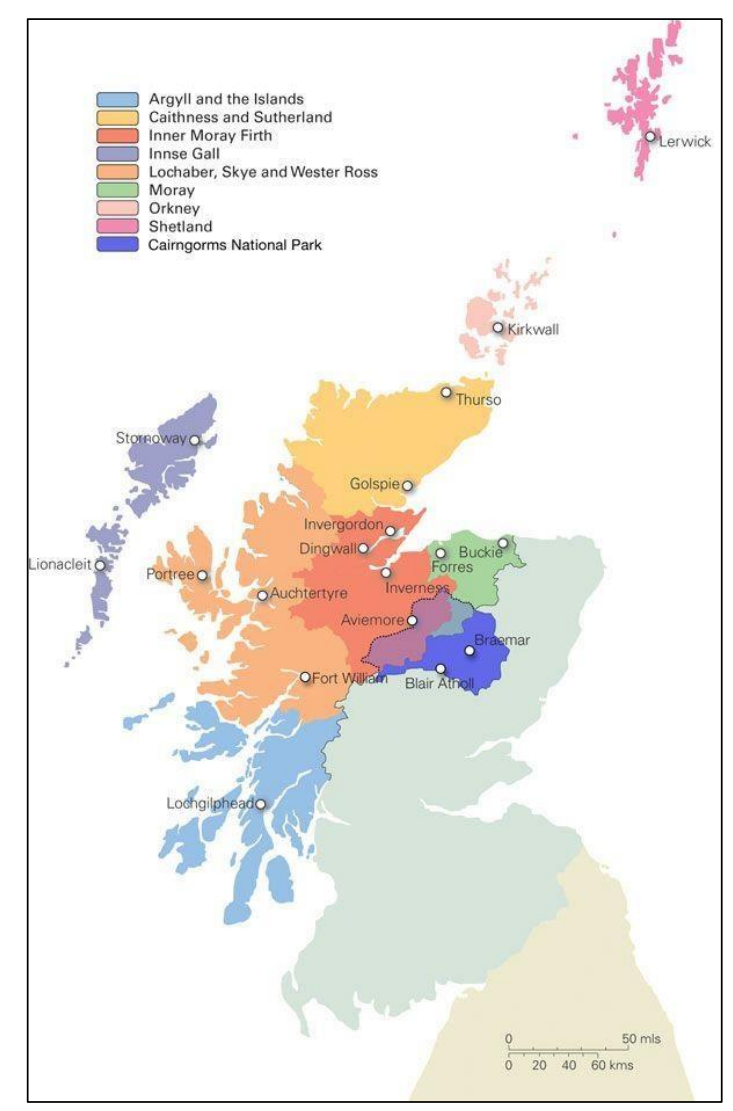

Fuente: SSPA (2017: Mapa 2).

El papel de la agencia en sus inicios fue principalmente el de llevar a cabo dos actuaciones: unir a los propietarios de las tierras y a los habitantes bajo una misma estrategia de desarrollo e incitar al gobierno británico a impulsar reformas legislativas que permitieran liberalizar el uso del suelo y facilitar su acceso a las comunidades locales. Paralelamente, desde el gobierno escocés se implementaron medidas que tuvieron una incidencia positiva en la región como la reforma del gobierno local en 1975 (reduciendo el número de municipios a seis) o la creación de un fondo de inversiones (proveniente de la explotación del petróleo del mar del Norte) para financiar proyectos de mejora de las infraestructuras de transporte (desde 1970 hasta 1990) y de las telecomunicaciones (desde 1990).

Así pues, con las reformas acometidas y la creación de la agencia, se apostó decididamente por desarrollar proyectos que fueran coste/efectivos dentro de sectores económicos con potencial y que resultaran de especial interés para la zona desde una perspectiva multidimensional, es decir, teniendo en cuenta aspectos económicos, sociales y medioambientales. Las actuaciones se centraron en dos sectores. En primer lugar, se invirtió en el sector pesquero tradicional, transformándolo en una industria conservera y de 
piscicultura moderna. En segundo lugar, se apostó por la mejora de la oferta turística mediante la valorización del patrimonio cultural y la profesionalización de los trabajadores, convirtiendo a esta región en un verdadero plató de cine y publicidad creando una imagen de marca territorial que fomenta los valores culturales y tradicionales de la región y que, como consecuencia, atrae a los visitantes y nuevos pobladores. Uno de los pilares del desarrollo de estos sectores ha sido la apuesta por las empresas de economía social con la creación un "ecosistema económico", en el que la agencia participa activamente diseñando un marco normativo estable, y que ha permitido que, con apenas el $8 \%$ de la población de Escocia, se concentren en la región el $22 \%$ de las empresas de economía social del país.

Junto a ello, otro de los principales proyectos ha sido la creación de una universidad (University of the Highlands and Islands) como herramienta para retener y atraer a jóvenes con talento, que cuenta con una oferta académica muy vinculada a las oportunidades del territorio y que es referente en la investigación a nivel internacional en sectores como las energías renovables marinas o la telemedicina, permitiendo asesorar y atraer a empresas foráneas y crear puesto de trabajo de calidad.

Así pues, todas estas iniciativas han conseguido cambiar la situación económica y social de esta región y ello se ha traducido en un giro en sus tendencias demográficas. Desde la creación de la agencia hasta la actualidad, las Tierras Altas e Islas han aumentado la población en un 22,4\% (desde 1961 hasta 2011), alcanzando cerca de medio millón de habitantes en la actualidad, mientras que el conjunto de la población en Escocia ha aumentado tan solo un 2,2\% (ver Figura 4). La autonomía técnica de la Agencia, que busca siempre la participación activa de los habitantes y está en coordinación con otras administraciones e instituciones, su planteamiento holístico, concibiendo el desarrollo del territorio de forma integral, junto con la financiación por parte del Estado, de la UE e incluso con sus recursos propios, han permitido que esta región se haya convertido en el ejemplo a seguir para otras regiones europeas, como las zonas mediterráneas más despobladas (SSPA), que vienen sufriendo un proceso de abandono demográfico y económico similar.

Gráfico 3. Evolución de la población en las Tierras Altas e Islas y en Escocia

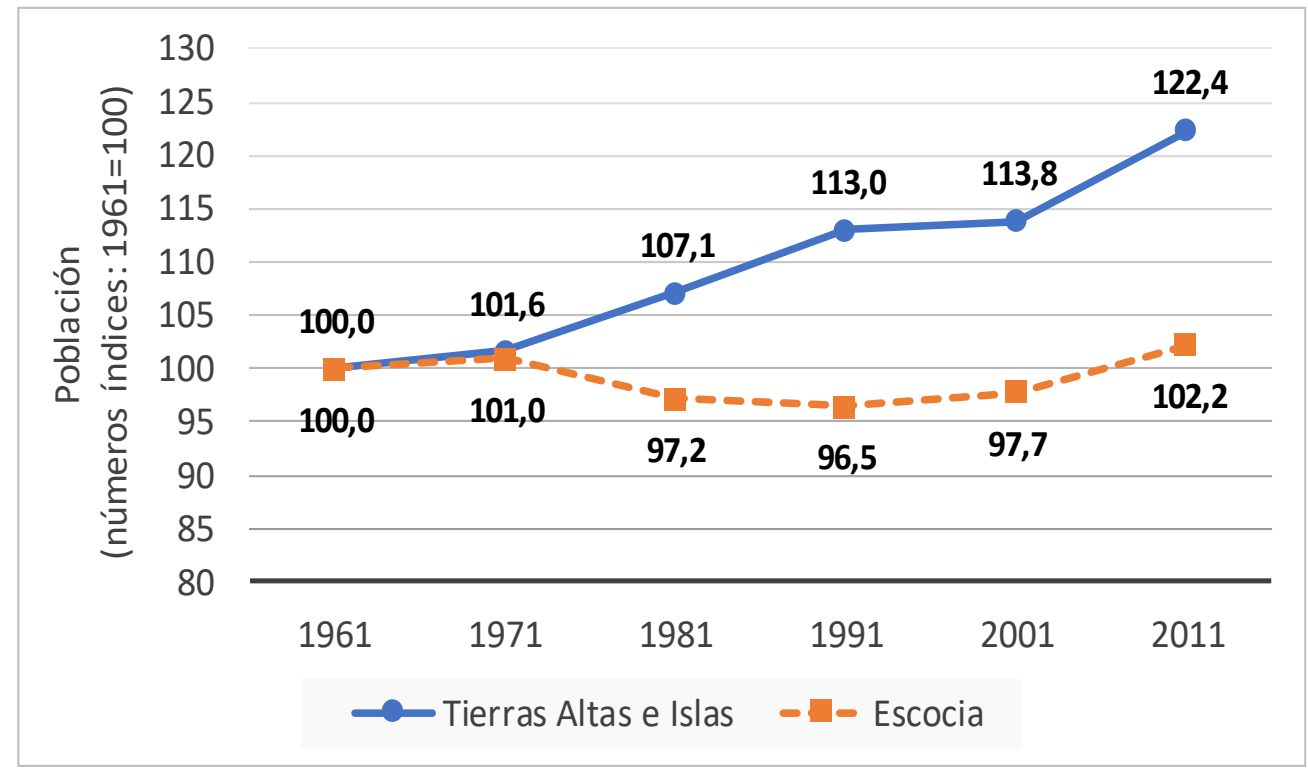

Fuente: elaboración propia a partir de SSPA (2017: Tabla 1). 


\subsection{Las denominaciones de origen e indicaciones geográficas protegidas en Francia e Italia y las granjas ecológicas en Austria}

La pérdida de ingresos que sufren los agricultores y la baja productividad de las explotaciones ganaderas son dos de los principales motivos por los que la agricultura y la ganadería tradicional están siendo abandonadas en las zonas rurales. Como consecuencia, el proceso de migración de jóvenes hacia las zonas urbanas, la pérdida de puestos de trabajo y el éxodo rural se está acelerando en muchos países de la UE y especialmente en España (Lasanta et al.,2005).

Sin embargo, a lo largo de la geografía europea existen ejemplos de políticas de desarrollo rural focalizadas en la recuperación de actividades agropecuarias basadas en la diferenciación y la calidad de los productos y ligadas al territorio bajo una dimensión ecológica y social (Bowen y Zapata, 2009). Entre estas actuaciones destaca principalmente la apuesta por la agricultura y la ganadería ecológica y los productos protegidos con denominaciones de origen, que permiten paralelamente el desarrollo de otras actividades alternativas como el ecoturismo, mercados, festivales, etc. (Darnhofer, 2005; Lorenzini, 2010). A continuación se presentan algunos de estos ejemplos.

Por lo que respecta a las denominaciones de origen e indicaciones geográficas protegidas, Francia e Italia fueron los países europeos precursores y cuentan con el mayor número de estos reconocimientos de calidad en sus productos agrícolas (Nathon, 2018). Mediante estas etiquetas se consigue proteger los derechos de propiedad intelectual y se evita la imitación de otros productores (principalmente de países terceros). Sin embargo, posiblemente, lo más importante es el aumento del precio que reciben los agricultores y ganaderos frente a otros productos debido a estas certificaciones de autenticidad y calidad. La Comisión Europea estima que los productos con Denominación de Origen (o Indicación Geográfica) Protegida más que duplican el precio de productos similares sin estas certificaciones (Nathon, 2018; DG AGRI, 2019a).

Algunos ejemplos que pueden servir de referencia de zonas que han apostado por estos certificados de calidad, con una visión integradora, sostenible e intrínseca al territorio, son el Valle d'Orcia en la Toscana y el área de Cinque Terre en Liguria, ambos casos en Italia, y la Alta Provenza en la región de Provenza-Alpes-Costa Azul en Francia.

Con respecto a los cinco municipios que forman parte del área de Cinque Terre, su actividad económica se basaba exclusivamente en la agricultura, la ganadería y, en el caso del único municipio con costa, también en la pesca. Como otras zonas rurales, venían sufriendo un fuerte proceso de despoblación como consecuencia de la falta de oportunidades laborales. Sin embargo, a partir de los años setenta se llevó a cabo un proceso de diversificación y transformación económica que mejoró notablemente su situación. En 1973 se creó una cooperativa vitivinícola y se consiguió la Denominación de Origen Protegida para los vinos de la zona con el consiguiente incremento en el precio de venta y los ingresos para los agricultores, lo cual permitió recuperar muchos puestos de trabajo (Lorenzini, 2010). Gracias a ello, en los años ochenta aumentó el ecoturismo y en 1999 se creó el Parque Nacional delle Cinque Terre para proteger el entorno. Así pues, aprovechando esta circunstancia, se crearon dos certificaciones (dentro del proyecto Presidia Slow Food ${ }^{7}$ ) para proteger la calidad y la tradición de una comida (sardinas de Montessoro) y un licor típico (sciaschetrà) de la zona y

\footnotetext{
${ }^{7}$ Este proyecto que nació en Italia y se ha extendido a otros países de Europa reconoce, mediante sellos de calidad, a los productos típicos y tradicionales de una zona en peligro de desaparición. Más información en: https://www.fondazioneslowfood.com/en/.
} 
una marca eno-gastronómica y de productos cosméticos bajo el nombre Parque Nacional delle Cinque Terre. Todo ello ha contribuido significativamente a aumentar el empleo de calidad por la creación de varias cooperativas de trabajo, dos laboratorios de investigación alimentaria y cosmética y un turismo sostenible respetuoso con el entorno (Lorenzini, 2010).

Por su parte, el Valle d'Orcia, en la Toscana italiana, destaca por sus dos Denominaciones de Origen Protegidas de vino (Brunello di Montalcino y Orcia). El primero (con DOP desde 1980) tiene un precio medio entre 20 y $200 €$ por botella, con ediciones especiales de vinos reserva que llegan a los $1.000 €$, mientras que el segundo (con DOP desde 2000 ) se vende entre 6 y $13 €$ (Lorenzini, 2010). Esta apuesta por productos locales de calidad ha permitido desarrollar paralelamente un enoturismo mediante el cual los propios agricultores han diversificado su actividad, transformando sus bodegas en alojamientos y vendiendo directamente su producción a los propios turistas. Con ello, se ha creado una conciencia social de pertenencia a la zona que ha incrementado la participación de las autoridades locales en la toma de decisiones (desarrollo endógeno) y una cultura de respeto por el entorno (sostenibilidad).

La Alta Provenza, en el sureste de Francia, se ha caracterizado desde el siglo XVIII por la producción de aceites esenciales. A finales de los años sesenta los productores locales se organización en cooperativas, lo que ayudó a la comercialización de estos productos. Tras este proceso surgieron en la zona numerosas empresas de alimentación y cosmética basadas en productos locales, entre las que destacó L'Occitane en Provence. El éxito internacional de esta empresa, junto con su reputación debida a la promoción de los productos naturales de la región, la responsabilidad social o la sostenibilidad (esta empresa fue pionera en reciclar los envases de sus productos) tuvo efectos positivos para la zona como destino turístico (Lorenzini, 2010). En 1981 la producción de aceite de lavanda consiguió la Denominación de Origen Protegida garantizando la calidad de sus productos a partir de estándares rigurosos obteniendo como recompensa unos mayores precios de venta y una diferenciación respecto a competidores de terceros países.

Por otro lado, en referencia a la agricultura y la ganadería ecológica, el caso objeto de estudio es el de Austria, país que cuenta con el mayor porcentaje de granjas y cultivos ecológicos (21,3\%) de Europa (MAPAMA, 2018) y con el mayor porcentaje de jóvenes ganaderos (el 22\% de los profesionales tienen menos de 40 años) según la Asociación Austriaca de Ganaderos (Campo Galego, 2019). La conversión o creación de granjas ecológicas tiene multitud de ventajas sobre la economía regional como el aumento del valor añadido de los productos, la protección del entorno o la posibilidad de venta directa de los productores, tal y como se ha demostrado en numerosos estudios (Knickel y Renting, 2000; Smith y Marsden, 2004). En este caso, los precios de venta pueden tener una prima del $25 \%$ hasta más que duplicar el valor de un producto similar no ecológico (DG AGRI, 2019b). Sin embargo, también es cierto que puede suponer una pérdida de producción para los agricultores y ganaderos que algunos estudios cifran en el $25 \%$ sobre la producción tradicional (EIP-AGRI, 2013).

La región de Baja Austria fue pionera en esta conversión ecológica y consiguió que otros sectores como el turístico, se sumasen a un desarrollo basado en el concepto de sostenibilidad, creando una imagen de marca ecológica que se extendió al resto de regiones de Austria. Todo esto permitió a los agricultores y ganaderos ganar poder de negociación en la cadena alimentaria al ofrecer productos diferenciados por su calidad y vender directamente 
a los consumidores (Zanoli, 2004). Junto a ello, el mayor supermercado del país (Billa/Rewe) creó una marca de productos ecológicos producidos en el país bajo el nombre de "Sí! Naturalmente/por supuesto" (Ja! Natürlich), lo que ayudó a mejorar su posicionamiento en el mercado. Paralelamente, se empezó a unir la marca de estos productos ecológicos con la sostenibilidad de los Parques Nacionales del país, con el nombre de "Eco-regiones" (Bioregionen), con el objetivo de promover el turismo rural en las granjas ecológicas diversificando los ingresos de los agricultores y ganaderos (Schermer, 2004). Así pues, mediante la agricultura y la ganadería ecológica se ha conseguido que veintidós regiones austriacas hayan apostado por un desarrollo sostenible, con una diversificación económica entre agricultura, ganadería, industria alimentaria ecológica, comercio de productos locales o turismo sostenible.

\subsection{El sistema de cooperativas agrarias en los Países Bajos y Dinamarca}

El proceso de apertura comercial llevado a cabo por la UE ha hecho que aumente la competencia de productos agrarios de terceros países generalmente con costes de producción más bajos. Ante esta situación, han sido habituales las protestas y manifestaciones de los agricultores y ganaderos de España y otros países del sur de Europa quejándose, en muchas ocasiones, de su escaso poder de influencia en la fijación del precio de sus productos y la asfixia económica a la que les someten los grandes distribuidores. Por el contrario, la incidencia de este problema en el norte de Europa es menor debido a los sistemas de cooperativas agrarias a través de las cuales los agricultores y ganaderos han aumentado su poder de negociación en la cadena de valor.

Por ejemplo, en los Países Bajos y Dinamarca existe una fuerte tradición de asociacionismo que ha permitido desarrollar grandes cooperativas con gran poder de influencia en el mercado. Por ejemplo, la cuota de mercado de las cooperativas en el sector agroalimentario de los Países Bajos es de un del 68\% (COGECA, 2019). La cooperativa más grande de los Países Bajos, FrieslandCampina (sector lácteo), emplea a cerca de 24.000 trabajadores, tiene 18.600 socios y una cifra de ventas superior 12.000 millones de euros (COGECA, 2019). En cuanto a Dinamarca, su mayor cooperativa, también del sector lácteo, es Arla Foods, que cuenta con más de 19.000 trabajadores, cerca de 12.000 miembros y una cifra de negocio de 9.600 millones de euros (COGECA, 2019).

La clave del éxito de las cooperativas de estos países reside, fundamentalmente, en cuatro factores: en primer lugar, su mayor tamaño y número de socios, que les permite tener una cuota de mercado más alta en sus respectivos sectores y ganar en eficiencia consiguiendo una reducción de los costes unitarios y rendimientos de escala (Bijman, 2016); en segundo lugar, su escasa y flexible legislación, que les permite adaptarse mejor a los cambios políticos y económicos del entorno y les da libertad para organizarse internamente en sus respectivos estatutos (Van der Sangen, 2013); en tercer lugar, una fuerte inversión en I+D+i, mediante el desarrollo de centros de investigación o contratos con Universidades, que tiene como resultado la creación de nuevos productos de mayor valor añadido y la diversificación de la oferta hacia productos con márgenes comerciales superiores a los productos tradicionales (Dobson, 2007); por último, el acceso a los canales de distribución mediante acuerdos comerciales con las grandes multinacionales ayuda a ampliar las fronteras y permite la internacionalización de su negocio y un crecimiento continuo (Juliá et a., 2012). 


\subsection{Las energías renovables en regiones rurales de Dinamarca, Suecia, Finlandia y Noruega}

Uno de los sectores económicos que puede jugar un papel destacado en el desarrollo de las zonas rurales es el de las energías renovables (Johnson y Altman, 2014). Según la OCDE (2012), en su informe "Linking Renewable Energy to Rural Development", las instalaciones para generar esta energía deben ubicarse en aquellos lugares donde se produzcan los procesos de obtención de estas fuentes renovables (agua, viento, sol, biomasa...) y que dispongan de espacio suficiente, motivo por el cual las zonas rurales con baja densidad de población se convierten en la localización más adecuada.

Sin embargo, dado que la energía renovable no es intensiva en mano obra, la mayor parte de los beneficios derivados de su explotación recaen sobre los propietarios de la tierra donde se instala y del capital que financia las infraestructuras energéticas (Bryden, 2010). En consecuencia, dado que la propiedad y el capital no suelen ser residentes en las zonas rurales (al contrario que los trabajadores), este sector puede convertirse en una oportunidad de desarrollo para estas zonas únicamente si se tiene en cuenta a los habitantes de estas comunidades locales como emprendedores, inversores y propietarios y no, únicamente, como meros espectadores de este desarrollo. Junto a ello, se requieren políticas e instrumentos públicos adecuados y suficientemente flexibles para adaptarse a las diferentes necesidades territoriales a fin de satisfacer el desarrollo del mercado energético y de las áreas locales donde se llevan a cabo estas actividades (Cavicci et al., 2014).

En países como Suecia, Dinamarca, Finlandia o Noruega se han aplicado, desde hace más de cuarenta años, políticas encaminadas a estos objetivos de manera que los gobiernos locales, junto con los habitantes de estas zonas rurales, como poseedores y administradores de tierras, han contribuido a generar beneficios para estos territorios en lugar de favorecer a inversores foráneos o grandes multinacionales (Midtunn y Koefoed, 2003; Breukers y Wolsink, 2007).

Un ejemplo concreto de este hecho es el caso de Dinamarca y el papel que han desempeñado los habitantes locales en el desarrollo de la energía eólica. Cuando el país decidió apostar por esta energía renovable (tras la crisis del petróleo de 1973) se dio preferencia a los residentes locales (principalmente agricultores y ganaderos) para que formaran cooperativas o para que pudieran adquirir participaciones en las infraestructuras para generar esta energía que se ubicaban en sus municipios. Para fomentar la participación de los habitantes locales en estos proyectos se obligó, a las empresas de servicios públicos, desde 1984, a garantizar el precio de compra de la energía eólica, pagando un $85 \%$ del precio minorista (Bryden, 2010). Con ello se consiguió una mayor implicación y desarrollo local y un reparto de los beneficios entre sus habitantes. Ahora Dinamarca se ha convertido en el primer país del mundo exportador de tecnología eólica y en 2018 el 46\% de la generación total de electricidad del país fue suministrada por esta energía renovable (Comisión Europea, 2020). Además, las empresas Vestas y Siemens Gamesa, principales productoras de aerogeneradores del mundo, tienen su sede en Aarhus y Brande respectivamente. Especialmente significativo es el caso de Brande, un pequeño pueblo de poco más de 7.000 habitantes de la región de Jutlandia Central, donde en los años ochenta se fundó la empresa eólica local Bonus, origen de la actual multinacional Siemens Gamesa.

Otro ejemplo a destacar, con respecto a la producción, distribución y comercialización de energía renovable, en este caso a partir de los recursos forestales, es el de Finlandia y Suecia. En ambos países, la energía de biomasa procedente de los recursos forestales es una 
parte integrada del sistema energético (principalmente en la industria y en el sector de la calefacción) y representa el 7,9\% y el 53\% respectivamente de la mezcla de combustible utilizado en calefacción urbana (Ericsson et al., 2004). La política energética general de ambos países ha apoyado a la biomasa mediante subsidios, principalmente para los sistemas de calefacción urbana (DHS) y la combinación de calor y energía. En el caso concreto de Suecia, la producción de este tipo de energía se benefició además de los impuestos a las emisiones de dióxido de carbono en la producción de calor que se establecieron en la década de los noventa. De manera que, aprovechando su riqueza forestal (Suecia es el primer país en masa boscosa de Europa), se incentivó la generación de esta energía renovable, principalmente en las zonas rurales, ayudando a crear nuevos puestos de trabajo más atractivos y mejor remunerados para los habitantes de estas zonas (Hillring, 2002). Al calor de estos incentivos nacieron numerosas empresas locales de producción de biomasa que con el tiempo han aumentado su tamaño (Briquette Energy es un ejemplo). De forma paralela la Asociación Nacional de Agricultores invirtió en pequeñas plantas de calefacción para que los agricultores pudieran explotarlas económicamente, mientras que en otros casos fueron los propios ayuntamientos los que crearon plantas de calor y electricidad. Incluso algunos ganaderos abandonaron su sector para dedicarse a la distribución, transporte y comercialización de productos relacionados con este tipo de energía, pasando de la fase de producción únicamente a otras partes de la cadena de valor (Hillring, 2002).

Por último, en el caso de Noruega, el $98 \%$ de la demanda de electricidad del país se cubre con energías renovables, de las que el $96 \%$ corresponde a la energía hidroeléctrica (IEA, 2020). El hecho diferencial en la producción de esta energía es que los ingresos derivados de ella corresponden, generalmente, a los habitantes de los municipios en las que se ubican las plantas hidroeléctricas. Aunque se trata en muchas ocasiones de micro plantas o de pequeña escala, estas son compartidas por pequeños grupos de agricultores o habitantes locales. Gracias a la Ley de Concesiones de licencias para la adquisición de las centrales hidroeléctricas de principios del siglo XX (Bryden, 2010), se consiguió distribuir los ingresos derivados de la explotación de estas instalaciones entre los municipios en los que se ubicaban, ayudando al desarrollo rural de estas zonas (Haakon et al., 2012). Hoy en día, aunque Noruega es el país de Europa que cuenta con mayores recursos naturales, como petróleo y gas, la apuesta decidida de sus autoridades por las energías renovables ha convertido al país en líder mundial en el uso de este tipo de energía.

\subsection{El turismo rural en Grecia e Irlanda: ejemplos de políticas endógenas, integradoras y sostenibles}

Tradicionalmente cuando se hablaba del turismo rural se destacaba su papel para fomentar y dinamizar de manera sostenible aquellas zonas rurales con especiales dificultades económicas y sociales por su efecto multiplicador sobre otras actividades (Flores y Barroso, 2012) y por su capacidad para generar puestos de trabajo (lakovidou, 2002). Además, según algunos estudios mediante el desarrollo de esta actividad se conseguiría frenar el proceso de despoblación (Alexander y McKenna, 1998; Nilsson, 1999) y se crearían puestos de trabajo que permitiría el desarrollo de estas zonas (Wanhill, 1977; OCDE, 1994; Comisión Europea, 2002).

No obstante, con el paso de los años se ha comprobado que la apuesta por este sector no ha conseguido, en la mayoría de casos, frenar el proceso de despoblación ni la crisis que vive el mundo rural (Rico, 2005; Cànoves, Villarino y Herrera, 2006; Sánchez y Sánchez, 2018). La principal explicación que se ha dado para este hecho es la falta de planificación en la puesta 
en marcha de proyectos relacionados con el turismo rural. En la mayoría de países las inversiones en turismo rural se han centrado en la construcción o rehabilitación de alojamientos sin llevar a cabo verdaderas políticas públicas coordinadas, endógenas, integradoras mediante la metodología bottom-up (de abajo-arriba) como la aplicada en la metodología Leader (Fleischer y Felsenstein, 2000).

A pesar de lo anterior, existen ejemplos en los que se han aplicado políticas de apoyo y desarrollo del turismo rural que han conseguido buena parte de los objetivos indicados. Entre estos ejemplos ya se han mencionado anteriormente el de las granjas ecológicas en Austria que han conseguido extender el concepto de sostenibilidad a otros sectores, incluyendo al turístico, o los casos de las regiones de Liguria y la Toscana en Italia en las que se ha incluido al turismo rural como una actividad más en la diversificación económica que se ha llevado a cabo.

Por otra parte, por lo que respecta a verdaderas políticas de apoyo al turismo rural, desde una perspectiva integradora de los habitantes de estas zonas, un ejemplo significativo es el que se lleva aplicando en Grecia desde comienzos de los años 80. Estas políticas se basaron en la puesta en marcha de programas de formación para el emprendimiento enfocados a mujeres que vivían en entornos rurales, facilitando la creación de cooperativas para el desarrollo del turismo rural (Koutsou et al., 2009). Desde el pequeño pueblo de Petra (en la Isla de Lesbos) en 1983, este movimiento se ha ido desarrollando en diferentes etapas, primero desde la Secretaría General por la Igualdad del Gobierno Heleno, posteriormente con la ayuda de los programas y proyectos europeos (NOW, EQUAL, LEADER) y finalmente con la Ley $2810 / 2000$ sobre las cooperativas que reducía el número mínimo de socios y permitió la creación de nuevas iniciativas relacionadas con el turismo rural bajo este tipo de organizaciones de economía social. Además, muchas autoridades locales han llevado a cabo iniciativas para ceder, en régimen de alquiler, edificios públicos vacíos para su uso comercial a este colectivo (Koutsou et al., 2009). Con todo ello, este tipo de políticas coordinadas y secuenciales en el tiempo constituyen la forma más genuina para el desarrollo de un verdadero turismo rural integrador, endógeno y sostenible, puesto que es implementado por un colectivo infrarrepresentado en el sector, que son residentes permanentes en las zonas rurales y con conexión con otras actividades como la agricultura, la gastronomía y los productos artesanales típicos de cada zona (lakovidou, 2002). Actualmente, este tipo de alojamiento rural aparece destacado como "cooperativas agroturísticas femeninas" en la página Web oficial de Turismo en Grecia, y se subraya que "al alojarte en ellas se contribuye en la promoción y la independencia económica de las mujeres en el medio rural".

Finalmente, otro ejemplo que permite hablar de un desarrollo sostenible e integrador del turismo rural lo representa el caso de Irlanda (Volkman, 2001; Heneghan, 2002) y más concretamente el de la región de Ballyhoura. El caso de esta región rural es especialmente significativo por la planificación que se ha llevado a cabo y la participación de la comunidad local para diversificar con éxito su economía desde una economía eminentemente agraria hacia el turismo rural. Para ello, se implementó una estrategia proactiva para la captación de fondos que se utilizaron para realizar investigaciones relacionadas con el desarrollo del turismo en la región (Robinson y O'Connor, 2013). Este tipo de investigaciones son imprescindibles para asegurar la aceptación e implicación de los habitantes antes de desarrollar estrategias y planes que les afecten directamente (lorio y Wall, 2012). 
En el caso de Ballyhoura se crearon dos organizaciones, Ballyhoura Development Limited (BDL) en 1989 y Ballyhoura Fáilte (BF) en 1986. BDL se estableció como una compañía de desarrollo local cuyo objetivo era apoyar a las personas y a las empresas a diversificar la económica de la localidad y mejorar la calidad de sus vidas a través del turismo. Por su parte BF se fundó como una cooperativa cuya función principal era actuar como operador turístico para captar visitantes (Robinson y O'Connor, 2012). Estas organizaciones estaban en coordinación con la política de desarrollo rural a nivel nacional y regional, a través de la asociación Teagasc, organismo nacional que brinda investigación, capacitación y asesoramiento a familias de agricultores, empresas rurales y comunidades rurales y que tiene como función clave la promoción del turismo rural como una opción viable para las comunidades agrarias (Teagasc, 2011).

Así pues, para llevar a cabo nuevos proyectos se creó una oficina de divulgación en cada uno de los cinco distritos de la región, cuyo Comité de Aprobación de Proyectos evalúa las solicitudes de financiación bajo los programas Leader, el Programa de Desarrollo Rural Nacional y el Programa de Inclusión Social a nivel local. Una vez recibidos los proyectos, el Comité considera la información proporcionada por el solicitante y hace una recomendación a la Junta para determinar la idoneidad del proyecto para recibir financiación pública (Robinson y O'Connor, 2013). A través de este proceso de coordinación se desarrollaron iniciativas locales para promover el turismo rural con una cuidadosa planificación estratégica y colaboración con los residente (Burns y Fridman, 2011) entre las que destacan: un Programa de Inclusión Social que imparte formación, soporte a los emprendedores, medidas de comercialización y apoyo al desarrollo de infraestructuras y productos relacionados con el turismo, una auditoría de los recursos e instalaciones turísticas disponibles en la zona, un análisis del perfil de los turistas para crear un manual de buenas prácticas que incidiera en la mejora de los servicios ofrecidos o la realización de una investigación para crear una infraestructura de ciclismo por montaña para atraer a visitantes extranjeros (Limerick County Development Board, 2012).

\section{Discusión: aplicabilidad en España de las diferentes estrategias}

Una vez explicadas de forma breve en el epígrafe anterior las cinco estrategias de desarrollo rural seleccionadas, en este realizaremos unas primeras consideraciones sobre su posible aplicación en España y los puntos clave a contemplar. Por otra parte, es necesario tener en cuenta que cada zona de las analizadas tiene sus características diferenciales propias, geográficas, culturales y socioeconómicas, por lo que no siempre se pueden aplicar fácilmente sus políticas a otras regiones, como las españolas, que tienen sus propias especificidades. Ahora bien, esto es un problema general de la economía e, incluso, de las ciencias sociales, ya que son de sobra conocidas las dificultades y cautelas necesarias para extrapolar comportamientos y políticas socioeconómicas de un territorio a otro y de un periodo temporal a otro.

Dependiendo de la estrategia, el reparto de responsabilidades entre el sector público y el privado es diferente, teniendo este último, posiblemente, más peso en los casos del cooperativismo y el turismo rural. Sin embargo, en todos los casos consideramos que el sector público es fundamental y que debería liderar todas las estrategias de desarrollo ya que, teniendo en cuenta la situación actual y el largo período de decadencia de la mayoría de las zonas rurales, es impensable asumir que dicha situación pueda ser revertida por el sector privado por sí solo: si hubiese podido hacerlo, ya lo habría hecho. 


\subsection{Agencias de desarrollo rural autónomas y despolitizadas}

Como ya hemos mencionado, el caso de las Tierras Altas e Islas de Escocia es un caso paradigmático de desarrollo rural en Europa. La característica más interesante y diferencial es la existencia de una autoridad autónoma y despolitizada, una agencia pública, para promover el desarrollo de la zona. Al no depender del ciclo político, es posible tener en cuenta en la planificación, no solo el corto plazo, sino también el medio y largo plazo, lo que permite mantener una línea coherente en el tiempo con beneficios acumulativos. Para poder llevar a cabo su objetivo, el desarrollo integral y sostenible de la región, la agencia dispone de autonomía financiera en el gasto y de autonomía técnica con profesionales cualificados incorporados mediante convocatorias públicas competitivas de empleo. Además, para mantenerse en contacto con la realidad económica y social de la región se le dio un peso fundamental a la participación pública con el plus de legitimidad que ello confiere.

En principio, no parece haber ninguna contraindicación para poder implantar agencias como esta en España. De hecho, podría haber una agencia de desarrollo rural en cada CC.AA. poniendo siempre en relación la población atendida con los recursos a disposición de la agencia y, quizás, una agencia estatal centrada en las labores de análisis comparado, comunicación interagencias o formación y prestación de servicios a las agencias autonómicas. Replicando la organización de Highlands and Islands Enterprise, cada agencia debería tener un consejo de administración con representantes estatales, autonómicos y locales vinculados al territorio, para asegurar la coherencia y coordinación con el resto de políticas económicas, pero la dirección operativa de la agencia debería recaer en cargos técnicos y no políticos. A pesar de las dificultades y resistencias que una medida de este tipo podría generar, puesto que algunas consejerías perderían competencias; debería ser posible puesto que es un tema de voluntad política y de reconocer que existen una serie de problemas comunes en todas las CC.AA. y similares a los existentes en otros países de Europa Occidental, que no han sido resueltos mediante las políticas económicas nacionales o regionales tradicionales. Es más, sería muy recomendable la existencia de agencias de desarrollo rural despolitizadas teniendo en cuenta la cada vez mayor crispación política existente.

\subsection{Denominaciones de origen, indicaciones geográficas y producción ecológica}

En cuanto a las etiquetas europeas de calidad (principalmente, Denominación de Origen, DOP o Indicación Geográfica Protegida, IGP) no hay duda de su contribución al valor de la producción del sector primario y de la industria agroalimentaria, como se ha comentado en los ejemplos seleccionados de Italia y Francia. De las más de tres mil certificaciones que hay de este tipo en la UE-28, España acumula el 11\% (ver Cuadro 1) y se sitúa en tercer lugar pero lejos de Italia y Francia que la duplican en número (DG AGRI, 2019a). Esta situación de privilegio en el ranking europeo es consistente con la importancia del sector primario español en la UE, el tercero en valor añadido bruto tras Francia e Italia y el primero de estos tres países en aportación del sector primario al PIB nacional (Eurostat, 2020). Sin embargo, en cuanto a los ingresos generados por las ventas de productos DOP/IGP certificados, España cae hasta el quinto puesto en términos de ventas totales y hasta el sexto en ventas medias por DOP/IGP, mientras que Francia e Italia mantienen los dos primeros puestos en volumen, no así en términos de ventas medias por DOP/IGP. Sin embargo, tanto por ser los lideres globales de este "sector", como por nuestra mayor similitud geográfica y afinidad sociocultural con estos dos países mediterráneos, nuestras referencias deben ser Francia e Italia. Las ventas medias 
de Francia por DOP/IGP duplican las de España e Italia, que tienen valores similares. Italia, por su parte, multiplica por 2,5 el número de DOP/IGP de España y, en consecuencia, también los ingresos totales. Por tanto, del análisis comparado con ambos países podemos concluir que España tiene todavía mucho potencial de crecimiento en este sector, especialmente en el comercio internacional a terceros países que está muy por debajo, no ya de Francia o Italia, sino de la media Europea.

Cuadro 1.Ventas 2017 de productos DOP/IGP de los principales Estados Miembros de la UE

\begin{tabular}{|l|c|c|c|}
\hline \multicolumn{1}{|c|}{ País } & $\begin{array}{c}\text { Ventas DOP/IGP 2017 } \\
\text { (millones } € \text { ) }\end{array}$ & No de DOP/IGP & $\begin{array}{c}\text { Ventas medias 2017 por } \\
\text { DOP/IGP (millones } € \text { ) }\end{array}$ \\
\hline Francia & 26.819 & 723 & 37,1 \\
\hline Italia & 15.758 & 847 & 18,6 \\
\hline Alemania & 8.672 & 162 & 53,5 \\
\hline Reino Unido & 7.698 & 68 & 113,2 \\
\hline España & 6.166 & 342 & 18,0 \\
\hline Otros Estados Miembros & 9.602 & 1006 & 9,5 \\
\hline Total UE & $\mathbf{7 4 . 7 1 5}$ & $\mathbf{3 . 1 4 8}$ & $\mathbf{2 3 , 7}$ \\
\hline
\end{tabular}

Fuente: elaboración propia a partir de (DG AGRI, 2019a: Tablas 1 y 46).

En cuanto a la agricultura ecológica ${ }^{8}$, España es el líder europeo en superficie cultivada, pero ocupa una posición media/baja en ganado, leches y productos lácteos ecológicos. Sin embargo, la penetración de estos productos entre los consumidores españoles e italianos es baja, alrededor del 3\% en 2017, por debajo del $5 \%$ alemán y el $4,4 \%$ francés y muy lejos del entorno del $10 \%$ que representan estos productos en Dinamarca o Suecia (DG AGRI, 2019b). Sin embargo, los crecimientos interanuales en su consumo son muy elevados ( $16 \%$ en España y $8 \%$ en Italia), por lo que también existe un potencial de futuro muy importante en este sector.

Teniendo en cuenta este potencial existente en España, que muestran las cifras de productos agrarios de alta calidad (DOP/IGP y ecológica), así como la capacidad de estos productos de generar externalidades positivas en otros sectores económicos (principalmente el turístico -ecoturismo o turismo sostenible, eno-gastronómico, agrario, etc.-, pero también hacia la industria alimentaria o incluso la investigación y el desarrollo) como hemos visto en los casos presentados de Italia, Francia y Austria, parece lógico que las políticas de desarrollo del mundo rural en nuestro país apuesten decididamente por este tipo de actuaciones.

\subsection{Cooperativas agrarias}

España es uno de los principales países europeos en número de cooperativas, socios y empleados como puede verse en el Cuadro 2. Sin embargo, como hemos visto en el epígrafe anterior, la cuestión clave es el tamaño. En los casos de España e Italia, el elevado número de cooperativas implica cierta atomización y unas ratios de ventas por cooperativa y por empleado significativamente inferiores a los de Alemania y Francia.

\footnotetext{
${ }^{8}$ Desde un punto de vista legal (Reglamento (CE) $n^{\circ} 834 / 2007$ del Consejo, de 28 de junio de 2007 , sobre producción y etiquetado de los productos ecológicos, http://data.europa.eu/eli/reg/2007/834/oj) los conceptos ecológico, biológico y orgánico son sinónimos. De hecho, en las diferentes traducciones del reglamento cada país utiliza el término con el que está más familiarizado: en España, utilizamos ecológico; en Francia, biológico; en el Reino Unido, orgánico y, en Alemania, ecológico/biológico.
} 
Cuadro 2. Principales datos del sector de cooperativas agroalimentarias en los países más grandes de la Unión Europea

\begin{tabular}{|l|c|c|c|c|}
\hline \multicolumn{1}{|c|}{ Estado miembro } & \multicolumn{1}{c|}{ Italia } & España & Francia & Alemania \\
\hline № de cooperativas & 4.703 & 3.755 & 2.400 & 2.024 \\
\hline № de socios & 742.078 & 1.150 .341 & 400.000 & 1.452 .000 \\
\hline № de empleados & 91.584 & 100.883 & 195.000 & 107.478 \\
\hline Ventas totales (millones $€$ ) & 34.969 & 28.993 & 84.400 & 62.100 \\
\hline Ventas medias por cooperativa (millones $€$ ) & 7,4 & 7,7 & 35,2 & 30,7 \\
\hline Ventas medias por empleado (miles $€$ ) & 382 & 287 & 433 & 578 \\
\hline
\end{tabular}

Fuente: elaboración propia a partir de COGECA (2109).

Si no solo nos comparamos con Alemania y Francia, sino que también lo hacemos con Dinamarca o los Países Bajos, naciones mucho más pequeñas que España y con un sector agrario menos potente que el nuestro en términos absolutos, la diferencia de tamaño medio es todavía más importante; y más aún si nos comparamos con las cooperativas más grandes de Europa, como puede verse en el Cuadro 3. En dicha tabla se muestran las cooperativas europeas con una cifra de ventas superior a cinco mil millones de euros junto con las cooperativas españolas más grandes, que apenas alcanzan los mil millones de euros de ventas. Incluso países mucho más pequeños que el nuestro, como los ya mencionados Países Bajos y Dinamarca, e incluso Finlandia, tienen cooperativas agroalimentarias más grandes que las españolas.

Cuadro 3. Cooperativas europeas y españolas con mayores volúmenes de ventas

\begin{tabular}{|c|c|c|c|c|c|c|}
\hline Cooperativa & País & Sector & $\begin{array}{l}\text { № de } \\
\text { socios }\end{array}$ & № de empleados & $\begin{array}{l}\text { Ventas totales } \\
\text { (millones } € \text { ) }\end{array}$ & $\begin{array}{c}\text { Ventas por } \\
\text { empleado (miles } € \text { ) }\end{array}$ \\
\hline BayWa & Alemania & Multisector & - & 17.550 & 16.050 & 915 \\
\hline FrieslandCampina & Países Bajos & Leche y lácteos & 18.645 & 23.675 & 12.110 & 512 \\
\hline Arla Foods & Dinamarca & Leche y lácteos & 11.922 & 19.190 & 9.600 & 500 \\
\hline DLG & Dinamarca & Cultivos herbaceos & 26.623 & 6.000 & 6.500 & 1.083 \\
\hline AGRAVIS Raiffeisen & Alemania & Agrario (general) & - & 6.685 & 6.400 & 957 \\
\hline DMK & Alemania & Leche y lácteos & 7.500 & 5.873 & 5.796 & 987 \\
\hline Invivo & Francia & Cultivos herbaceos & - & 11.400 & 5.500 & 482 \\
\hline Agrial & Francia & Agrario (general) & 13.000 & 22.000 & 5.495 & 250 \\
\hline Terrena & Francia & Agrario (general) & - & 15.754 & 5.141 & 326 \\
\hline Sodiaal & Francia & Leche y lácteos & 111.764 & 9.100 & 5.068 & 557 \\
\hline Metsä Group & Finlandia & Forestal & 103.752 & 9.126 & 5.040 & 552 \\
\hline DCOOP (Andalucía) & España & Aceite de oliva & 85.482 & $* 576$ & $* 1.230$ & 1.925 \\
\hline Coren (Galicia) & España & Ganadería & 4.739 & 3.240 & $* 1.078$ & 309 \\
\hline Grupo AN (Navarra) & España & Agrario (general) & - & $* 1.289$ & $* 1.074$ & 833 \\
\hline
\end{tabular}

Fuente: elaboración propia a partir de COGECA (2019) y OSCAE (2019). Los datos de España con * provienen de OSCAE.

Asociado a estos mayores tamaños están unas mayores cuotas de mercado en sus respectivos sectores y un poder de negociación mucho más elevado que el que tienen las cooperativas españolas. Si los agricultores y ganaderos españoles se organizaran en cooperativas de mayor tamaño que las actualmente existentes se podrían solucionar, parcialmente al menos, algunos de los problemas del campo español (Juliá et al., 2012). De hecho, esta mayor concentración en el sector de las cooperativas y el consiguiente incremento 
de su tamaño, se encuentra entre las tendencias estructurales del sector, especialmente entre las más grandes (OSCAE, 2019).

Por tanto, entre las políticas de desarrollo rural que debe adoptar España no se debe olvidar el cooperativismo, promoviendo nuevas cooperativas donde no las haya y, sobre todo, fomentando la integración de las existentes. Una mayor dimensión permitirá en primer lugar, una mayor influencia en el reparto de beneficios a lo largo de la cadena de distribución, mejorando la situación económica de los agricultores y ganaderos y de los territorios rurales en los que se asientan y, en segundo lugar, permitirá abordar con éxito o con mayor éxito aun, los procesos de internacionalización e $1+D+i$ que son claves para el desarrollo de las cooperativas agroalimentarias, tal y como hemos visto en las experiencias analizadas en el epígrafe anterior.

\subsection{Energías renovables en zonas rurales}

Durante la primera década del siglo XXI (2000-2010), España fue uno de los líderes europeos y mundiales en instalación de energías renovables. A nivel mundial, solo nos superaban, de mayor a menor, China, Alemania, Estados Unidos, India y Brasil ${ }^{9}$. El más pequeño de estos países (Alemania) casi nos duplica en población. En la década siguiente (2010-2020), la crisis económica, el recorte a las primas o subvenciones a las renovables y, finalmente, el "impuesto al sol"10, redujeron a la mitad la instalación de renovables en España cuando en el mundo se multiplicó por tres, perdiendo nuestra posición de liderazgo europeo y mundial.

Sin embargo, a pesar de las experiencias comentadas del norte de Europa, algunas de ellas realmente antiguas, este ciclo de inversión, de aproximadamente 15 años, no ha sido aprovechado para contribuir al desarrollo rural de una forma planificada o sistemática. Sí que ha habido inversiones en el mundo rural, ya que es donde había espacio para situar las instalaciones renovables, pero las inversiones han corrido a cargo de las empresas energéticas, especialmente en energía eólica, y en consecuencia, la mayor parte de los beneficios también han sido para ellas y no se han quedado en el territorio de generación de la energía renovable. Indudablemente, la utilización de los espacios rurales para instalar las plantas solares o las turbinas eólicas ha aportado unos ingresos considerables a los municipios donde se han instalado en forma de compras o alquileres de terrenos, empleos en su construcción y mantenimiento o recaudación de impuestos (Galdós y Madrid, 2009). No obstante, en primer lugar, este sector es intensivo en capital y no en mano de obra y, en segundo, se ha podido generar cierto "rentismo" por parte de los municipios y particulares que alquilaban los terrenos. Hay que tener en cuenta que, mientras que los puestos de trabajo sí que fijan a la gente al territorio, las rentas no necesariamente, puesto que se pueden seguir percibiendo aunque la población rural se traslade a la ciudad. Por tanto, en muchas ocasiones, las millonarias inversiones no han modificado la actividad productiva de la zona, que sigue

\footnotetext{
${ }^{9}$ Elaboración propia a partir de datos de IRENA (2020).

${ }^{10} \mathrm{El}$ "impuesto al sol" fue un tributo para aquellas instalaciones fotovoltaicas que generasen energía eléctrica para autoconsumo y estuviesen conectadas a la red general. Estas instalaciones, a no ser que estuviesen aisladas, debían pagar impuestos y peajes por toda la electricidad generada en la instalación, incluso la producida y consumida en la propia instalación. De esta forma contribuirían a sufragar los costes del sistema y no se reducirían los ingresos y márgenes de los operadores energéticos tradicionales. La consecuencia de este impuesto fue un descenso de la rentabilidad de las instalaciones fotovoltaicas que, acumulado a la reducción de las primas, produjo una paralización casi absoluta de nuevas instalaciones. Dicho impuesto fue aprobado por el Real Decreto 900/2015 y derogado mediante el Real Decreto-Ley 15/2018.
} 
dependiendo del sector primario, pudiendo existir, incluso, cierta sensación entre los ayuntamientos de que los beneficios económicos no son suficientes (IDAE, 2006).

De todos modos, hay casos de todo tipo con excepciones positivas y negativas, especialmente entre aquellos municipios que disponen de rentas extraordinarias debido a una elevada concentración de aerogeneradores. Por ejemplo, en Ampudia, municipio palentino de 600 habitantes, los más de 130 aerogeneradores (más de $230 \mathrm{MW}$ de potencia instalada) han proporcionado unos elevados niveles de ingresos que han permitido rehabilitar el núcleo urbano y el patrimonio histórico artístico, fomentando el turismo y logrando así una diversificación económica (Morales y Herrero, 2013). Como ejemplo negativo podemos mencionar el de La Muela, pueblo zaragozano de mil habitantes en 1990 que fue uno de los primeros en instalar aerogeneradores en España a finales de los años 80. En la actualidad el municipio, de 5.000 habitantes, ingresa unos $2.000 €$ al año por cada uno de los 500 aerogeneradores instalados en el término municipal. Sin embargo, el dinero recibido de los aerogeneradores se despilfarró principalmente en gasto suntuario no productivo (museos, plaza de toros, conciertos con artistas famosos, equipamientos deportivos no necesarios, etc.) y en tramas de corrupción urbanística (Martín, 2009).

A pesar de haber desaprovechado la burbuja inicial de las renovables y de casos como el de La Muela, la lucha contra el cambio climático y la decidida apuesta de las autoridades europeas y españolas por "ecologizar" la economía, hace imprescindible seguir apostando por estas fuentes de energía para diversificar la economía en las zonas rurales. De hecho, es muy probable que la UE en el futuro preste más atención a la relación entre energías renovables y desarrollo rural, ya que el Tribunal de Cuentas Europeo (2018) ha advertido que la política europea de desarrollo rural no está teniendo en cuenta suficientemente las sinergias existentes entre estos dos campos.

No obstante, para que estas inversiones puedan llegar a modificar de forma efectiva la estructura productiva, diversificando la economía de estas zonas, es necesario aplicar las lecciones extraídas de los casos de éxito del norte de Europa. La principal lección que podemos extraer es que es necesario que las inversiones sean realizadas por los propios habitantes rurales, de forma que las renovables se conviertan en una actividad económica de la que son propietarios en vez de una mera renta. De esta forma, la mayor parte de los beneficios generados, no solo el trabajo, sino también las rentas de la tierra o los beneficios empresariales se quedarán y revertirán en el propio territorio rural.

Esto solo puede hacerse con un decidido apoyo del sector público y, una forma lógica de hacerlo es fomentando las cooperativas energéticas. Aunque estas cooperativas tienen una larga tradición en nuestro país, tampoco aprovecharon el auge de las renovables en el siglo XXI en un primer momento y, a partir de la crisis económica de 2008 , la rentabilidad de las renovables decayó de forma notable y prácticamente desapareció a partir de 2012 debido a la "obstrucción a las fuentes de energía renovables" (Capellán-Pérez et al., 2018) realizada por el gobierno con el apoyo del sector eléctrico español. Aunque la mayoría de cooperativas surgidas en los últimos años son comercializadoras, ya que el capital inicial es mucho menor que para generación de energía renovable, poco a poco van consiguiendo activos de generación modestos (Capellán-Pérez et al., 2018). Entre las cooperativas que tienen capacidad de generación podríamos mencionar a Som Energía (Cataluña), Energética (Castilla y León), Barrizar o Goiener (País Vasco). Aunque estas cooperativas hayan sido creadas, principalmente, en entornos urbanos, las instalaciones renovables suelen estar localizadas en 
entornos rurales y, hoy en día, la tecnología y las comunicaciones permiten alcanzar una masa crítica de personas para poner en marcha cooperativas similares en las zonas rurales.

\subsection{El turismo rural}

El turismo rural es la actividad más utilizada para diversificar la economía en las zonas rurales ya que, en principio reunía todas las condiciones necesarias para convertirse en el complemento perfecto para el desarrollo rural. Sin embargo, como ya hemos mencionado, aunque ha contribuido a mejorar económicamente la situación del campo en España creando empleo (Sánchez y Sánchez, 2018) y rentas complementarias (Del Valle, 2015), no ha logrado evitar la despoblación del mundo rural (Maroto y Pinos, 2019).

Ahora bien, aunque el turismo rural no haya sido capaz de solucionar por sí mismo los problemas del campo español, convirtiéndose en su principal sector económico, en prácticamente todos los casos que hemos mencionado hemos podido comprobar que el turismo era una actividad complementaria fundamental: en el caso escoces se promocionó la región desde el punto de vista turístico, las DOP/IGP y las granjas ecológicas también fomentaron el turismo rural asociado a sus productos e, incluso, las rentas de las energías renovables se utilizaron para rehabilitar Ampudia y fomentar el turismo.

Por tanto, y teniendo en cuenta que las posibilidades de diversificación económica del entorno rural son limitadas, consideramos imprescindible seguir apostando por el turismo rural. Las experiencias en Grecia e Irlanda, así como numerosas experiencias españolas en las que aquí no podemos entrar, nos demuestran que todavía este sector tiene mucho recorrido para contribuir al desarrollo rural.

\section{Conclusiones}

En este artículo se han explicado de forma breve cinco estrategias exitosas que han contribuido a solucionar en sus regiones, aunque no sea de forma completa, gran parte de los problemas que actualmente tienen las zonas rurales de Europa Occidental. Adicionalmente, se ha realizado un primer análisis de la posibilidad de aplicar o profundizar en dichas estrategias en España.

La conclusión obtenida es que todas ellas son aplicables en nuestro país y, de hecho, todas ellas pueden implementarse, incluso, de forma simultánea, ya que no son excluyentes. Por ejemplo, la dirección de las políticas de desarrollo rural en cualquier lugar podría recaer sobre una agencia de desarrollo rural independiente. Esta agencia podría optar por fomentar las DOP/IGP y la producción ecológica y crear cooperativas agroalimentarias. Ambas medidas se complementarían perfectamente ya que, mediante productos de más calidad y un mayor poder de negociación en la cadena de distribución, se conseguiría una retribución más alta para los agricultores y ganaderos. La promoción de cooperativas no tendría por qué limitarse a las agroalimentarias ya que la experiencia acumulada podría aprovecharse para la creación de cooperativas de energías renovables. Por último, el turismo rural podría promocionarse como una actividad complementaria a todas las demás.

Un mundo rural con una economía diversificada basada en un sector primario de alta calidad (DOP/IGP y ecológico) y un sector secundario de cooperativas de energías renovables, ambos organizados mediante cooperativas locales, complementado por un sector terciario apoyado en el turismo, estaría en mejores condiciones para ofrecer a sus habitantes oportunidades de futuro evitando la despoblación. Lograr estos objetivos a medio y largo es 
posible que fuese más sencillo si todo fuese dirigido y coordinado por una agencia de desarrollo rural autónoma.

Hay experiencias suficientes en España y en Europa y, por tanto, herramientas ya testadas, para tratar de mejorar la situación del mundo rural. Es el sector público, mediante sus políticas, el que debe tomar las riendas y demostrar voluntad para tratar de solucionar los problemas de este importante sector de la población.

\section{Bibliografía}

Alexander, N. y Mckenna A. (1998).Rural tourism in the Heart of England. International Journal of Contemporary Hospitality Management, 10 (5), 203-207.

Bahadillo, C. (2005). Crecimiento económico y desarrollo sostenible en el medio rural ¿Utopía o realidad? Revista de Desarrollo Rural y Cooperativismo agrario, 9, 9-20.

Bijman, J. (2016). Agricultural Cooperatives in the Netherlands: Key Success Factors. Cooperatives: the power to Act. International Summit of Cooperatives. Quebec.

Bijman, J., Iliopoulos, C., Poppe, K. J., Gijselinckx, C., Hagedorn, K., Hanisch, Hendrikse, G. W. J., Kühl, R., Ollila, P., Pyykkönen P., y v. d. Sangen, G. (2012). Support for Farmers' Cooperatives: Final Report. Wageningen, The Netherlands, Wageningen UR. Disponible en: http://edepot.wur.nl/245008.

Bowen, S. y Zapata, A.V. (2009). Geographical indications, terroir and socioeconomic and ecological sustainability: The case of Tequila. Journal of rural studies, 25, 108-119.

Breukers, S. y Wolsink, M. (2007). Wind power implementation in changing institutional landscapes: An international comparison. Energy Policy, 35, 2737-2750.

Bryden, J. (2010). Renewable Energy as a Rural Development Opportunity. Norwegian Agricultural Economics Research Institute (NILF). Discussion Paper no8.

Burns, P. y Fridman, D. (2011). Actors' Perceptions of the Newly Designated South Downs Park: Social Exchange Theory and Framework Analysis Approach. Tourism Planning and Development, 8 (4), 447-465.

Campo Galego (2019). Austria es el país de la Unión Europea con mayor porcentaje de jóvenes en la ganadería y agricultura, Campo Galego. Xornal dixital agrario, 2/10/2019. Disponible en: https://www.campogalego.es/austria-es-el-pais-de-la-union-europeacon-mayor-porcentaje-de-jovenes-en-la-ganaderia-y-agricutlura/

Cànoves, G., Villarino, M. y Herrera, L. (2006). Políticas públicas, turismo rural y sostenibilidad. Boletín de la Asociación de Geógrafos Españoles, 41, 199-220.

Capellán-pérez, I., Campos-celador, A., Terés-zubiaga, J. (2018). Renewable Energy Cooperatives as an instrument towards the Energy Transition in Spain. Energy Policy, 123, 215-229. Disponible en: https://doi.org/10.1016/j.enpol.2018.08.064.

Cavicci, B., Bryden, J. y Vittuari, M. (2014). A comparison of bioenergy policies and institutional frameworks in the rural areas of Emilia Romagna and Norway. Energy Policy, 67, 355-363.

COGECA (2019). The European agri-food cooperatives monitor 2019. CCC (19) 9923:2.

Comisión Europea (2002). Ireland's Experience of Regional Development Working Document (ESC side). Fifth Meeting of the EU-Poland Joint Consultative Committee, Varsovia 1114 Mayo. 
Comisión Europea (2020). EU energy in figures. Statistical pocketbook 2020. Luxembourg: Publications Office of the European Union. Disponible en: https://op.europa.eu/s/osjs Darnhofer, I. (2005). Organic Farming and Rural Development: Some Evidence from Austria. Sociologia Ruralis, 45 (4), 308-323.

De Pablo, J. y Mirta M. E. (2002). Política de desarrollo rural en países desarrollados y países en desarrollo en Lledó, F. y Herrera, R. (Coords.): Agricultura ante el tercer milenio. Dykinson.

Del Valle, E. A. (2015). El turismo rural en España. Análisis de la evolución del modelo de desarrollo y perspectivas futuras (Tesis doctoral). Universidad de Vigo. Disponible en: http://www.investigo.biblioteca.uvigo.es/xmlui/bitstream/handle/11093/632/El_tur ismo_rural_en_Espa\%C3\%B1a.pdf?sequence=1\&isAllowed=y

DG AGRI (2019a). Study on economic value of EU quality schemes, geographical indications (GIs) and traditional specialities guaranteed (TSGs). Final Report. Comisión Europea, Directorado-General para la Agricultura y el Desarrollo Rural. Luxembourg: Publications Office of the European Union. Disponible en: https://op.europa.eu/s/os3V

DG AGRI (2019b). Organic farming in the EU. A fast growing sector. EU Agricultural Markets Briefs No 13, March 2019. Comisión Europea, Directorado-General para la Agricultura y el Desarrollo Rural. Luxembourg: Publications Office of the European Union. Disponible en: https://ec.europa.eu/info/sites/info/files/food-farmingfisheries/farming/documents/market-brief-organic-farming-in-theeu_mar2019_en.pdf

Dobson, W. (2007). Competitive strategies of dairy processing firms in Ireland. Babcock Institute Discussion Paper, 3.

EIP-AGRI (2013). Focus Group-Organic Farming, Optimising Arable Yields. Recommendations and Outputs. European Innovation Partnership (EIP-AGRI) Agricultural Productivity and Sustainability. Comisión Europea.

Energy International Agency (2020). Data and Statistics. Disponible en: https://www.iea.org/data-andstatistics?country=NORWAY\&fuel=Renewables\%20and\%20waste\&indicator=Hydroel ectric\%20electricity\%20generation

Ericsson, K., Huttunen, S., Nilsson, L . J. y Svenningsson, P. (2004). Bioenergy policy and market development in Finland and Sweeden. Energy Policy, 32, 1707-1721.

Esparcia, J. (2019). "Prohibido teorizar": del diagnóstico a la acción en la lucha contra el despoblamiento rural. Reptes de la vertebració territorial valenciana. ISBN 9788491332237, 55-69.

ESPON (2017). Shrinking rural regions in Europe. Disponible en: https://www.espon.eu/ruralshrinking

Eurostat (2016). Urban Europe - Statistics on Cities, Towns and Suburbs - Patterns of Urban and City Developments - Statistics Explained. Disponible en:

http://ec.europa.eu/eurostat/statistics-

explained/index.php/Urban_Europe_\%E2\%80\%94_statistics_on_cities,_towns_and_ suburbs_\%E2\%80\%94_patterns_of_urban_and_city_developments

Eurostat (2019a). Methodology manual on territorial typologies - Statistics Explained. Disponible en: https://ec.europa.eu/eurostat/statisticsexplained/index.php?title=Territorial_typologies_manual__degree_of_urbanisation\#Classes_for_the_typology_and_their_conditions 
Eurostat (2019b). Eurostat regional yearbook 2019 edition. Libros de Estadísticas. ISBN 97892-76-03505-3. Oficina de Publicaciones de la Unión Europea. Luxemburgo.

Eurostat (2020). Gross value added and income by $A^{*} 10$ industry breakdowns [NAMA_10_A10]. Eurostat Database. Disponible en:

https://ec.europa.eu/eurostat/databrowser/view/NAMA_10_A10/default/table?lan $\mathrm{g}=\mathrm{en}$

Fleischer, A. y Felsenstein, D. (2000). Support for rural tourism: does it make a difference? Annals of Tourism Research, 27 (4), 1007-1024.

Flores, D. y Barroso, M. (2012). El turismo como estrategia de desarrollo rural sostenible. Los parques naturales andaluces. Revista de Estudios Empresariales, 1, 59-83.

Frutos, L. M. (2006). Problemas y perspectivas del mundo rural en Frutos, L. M. a y Ruiz, E. (eds.), Estrategias Territoriales de Desarrollo Rural. Ed. Institución Fernando El Católico. Zaragoza.

Galdos, R. y Madrid, F. J. (2009). La energía eólica en España y su contribución al desarrollo rural, Investigaciones Geográficas, 50, 93-108

Haakon, T., Hakon, S. y Atlet H. (2012). Development of small versus large hydropower in Norway - comparison of environmental impacts. Energy Procedia, 20, 185-199.

Heneghan, M. (2002). Structures and processes in rural tourism. Paper presented at the Rural Development Conference: Signposts to Rural Change, Tullamore Court, Teagasc, Dublin, February.

Hillring, B. (2002). Rural development and bioenergy - experiences from 20 years of development in Sweeden. Biomass and Bioenergy, 23, 443-451.

lakovidou, O. (2002): Women's agrotourist cooperatives in Greece: key elements for their successful operation. Journal of Rural Cooperation, 30 (1), 13-24.

IDAE (2006). Energía eólica, Manuales de Energías Renovables, 3. Madrid: Instituto para la Diversificación y Ahorro de la Energía (IDAE). Disponible en:

http://www.idae.es/uploads/documentos/documentos_10374_Energia_eolica_06_2 e6a15a7.pdf

Ilbery, B., Morris, C., Buller, H., Maye, D. y Kneafsey, M. (2005). Product, Process and Place. An Examination of Food Marketing and Labelling Schemes in Europe and North America. European Urban and Regional Studies, 12 (2), 116-132.

Iorio, M. y Wall, G. (2012). Behind the Masks: Tourism and Community in Sardinia. Tourism Management, 33(6), 1440-1449.

IRENA (2020). Renewable Electricity Capacity and Generation Statistics, July 2020. International Renewable Energy Agency (IRENA). Disponible en:

https://www.irena.org/Statistics/Download-Data

Jiménez, F. J. (2000). Turismo rural y empleo. VII Congreso de Economía Regional de Castilla y León. Soria.

Johnson T. y Altman, I. (2014). Rural development opportunities in the bioeconomy. Biomass and Bioenergy, 63, 341-344.

Juliá, J. F., García, G. y Meliá, E. (2012). La Globalización y los Modelos de Crecimiento de los Grupos Cooperativos: las Cooperativas Agroalimentarias en España y en la Unión Europea. Ekonomiaz, 79, 1r cuatrimestre de 2012.

Knickel, K. y Renting, H. (2000). Methodological and conceptual issues in the study of multifunctionality and rural development. Sociologia Ruralis, 40 (4), 512-528. 
Koutsou, S., Notta, O., Samathrakis, V. y Partalidou, M. (2009). Women's Entrepreneurship and Rural Tourism in Greece: Private Enterprises and Cooperatives. South European Society and Politics, 14 (2), 191-209.

Lacambra, V. M. (2001). Desarrollo rural en los espacios rurales europeos. Elementos de desigualdad territorial. Revista Catalana de Sociologia, 14, 253-276.

Lasanta, T., Vicente, S. M. y Cuadrat, J. M., (2005). Mountain Mediterranean landscape evolution caused by theabandonment of traditional primary activities: a study of the Spanish Central Pyrenees. Applied Geography, 25(1), 47-65.

Limerick County Development Board (2012). Development Plans. Disponible en: https://www.limerick.ie/council/services/planning-and-property/developmentplans/county-development-plan

Lorenzini, E. (2010). Origin Labelled Products, Territorial Marks and their contribution to Rural Development. Evidence from Italy and France. Società Italiana di economia pubblica, no 649 .

MAPAMA (2018). Estrategia para la producción ecológica 2018-2020.

Maroto, J. C. y Pinos, A. (2019). ¿El Turismo Rural freno a la despoblación? El caso del sur de España, en Cejudo, E. y Navarro, F. (Ed.) (2019): Despoblación y transformaciones sociodemográficas de los territorios rurales: los casos de España, Italia y Francia.

Midttun, A. y Koefoed, A. L. (2003). Green Innovation in Nordic Energy Industry: Dinamic Patterns and Institutional Trajectories. Paper for the Conference: "Innovation in Europe: Dynamics, Institutions and Values". Roskilde University, Denmark, 8th-9th May.

Martin, A. (2009). La Muela, el pueblo que vive del aire, RTVE, 18/03/2009. Disponible en: https://www.rtve.es/noticias/20090318/muela-pueblo-vive-del-aire/249336.shtml

Morales, E. y Herrero, D. (2013). La contribución de la energía eólica al desarrollo rural en Ampudia en Universitat de les Illes Balears, Espacios insulares y de frontera, una visión geográfica, Congreso de Geógrafos Españoles, 23-25/10/2013, Palma de Mallorca. Palma: Universitat de les Illes Balears, pp. 631-640.

Nathon, N. (2018). Geographical Indications in the EU. Agriculture and Rural Development. Comisión Europea. Tel Aviv.

Nilsson P. (1999). Tourism's role in a new rural policy for peripheral areas: the case of Archipelago en Brown, F. y Hall, D. (eds). Case Studies of Tourism in Peripheral Areas, Bornholms Forskningscenter: Nexø; pp.157.180.

OCDE (1994). Tourism Strategies and Rural Development. Organization for Economic Cooperation and Development. Paris.

OCDE (2012). Linking Renewable Energy to Rural Development. OECD Publishing, (OECD Green Growth Studies). Paris. Disponible en:

http://dx.doi.org/10.1787/9789264180444-en

OSCAE (2019). El cooperativismo agroalimentario español. Informe socioeconómico 2018. Observatorio Socioeconómico del Cooperativismo Agroalimentario Español, Cooperativas Agro-alimentarias de España. Disponible en: http://www.agroalimentarias.coop/cooperativismo_en_cifras

Pezzini, M. (2001). Rural Policy Lessons From OECD Countries. International Regional Science Review, 24 (1), pp. 134-145.

Plaza, J. I. y Araque, E. (2000). El turismo rural como fuente de desarrollo sostenible: best practices. Revista de Economía y Finanzas de Castilla y León, 4, 77-100. 
Reig, E., Goerlich, F. J. y Cantarino, I. (2016). Delimitación de áreas rurales y urbanas a nivel local. Demografía, coberturas del suelo y accesibilidad. ISBN: 978-84-92937-64-6. Fundación BBVA. Bilbao.

Rico, M. (2005). El turismo como nueva fuente de ingresos para el medio rural de Castilla y León. Cuadernos de Turismo, 16, 175-195.

Robinson, J. y O'Connor, N. (2012). Ballyhoura - A Case Study of Cohesive Rural Tourism Planning in Ireland. International Conference on Tourism and Events: Opportunities, Impacts and Change (Incorporating the 8th Annual Tourism and Hospitality Research in Ireland Conference), Ulster Business School, University of Ulster, Belfast, Northern Ireland.

Rodríguez, R. y Pérez, E. (coord.) (2004). Espacios y desarrollos rurales: una visión múltiple desde Europa y Latinoamérica. Ediciones TREA, Gijón.

Rubio, P. (1997). La crisis del mundo rural: experiencias de desarrollo en la provincia de Teruel. Publicaciones de la Universidad de Zaragoza. Zaragoza.

Sánchez, A. M. y Sánchez F. J. (2018). Impacto del turismo rural sobre el empleo en España: una aproximación a escala provincial. Cuadernos de Desarrollo Rural, 15 (82).

Schermer, M. (2004). The concept of Eco-regions in Austria en Farming and rural systems research and extension. Pre-Proceedings of the 6th European Symposium of the International Farming Systems Association. Vila Real (Portugal).

Smith, E. y Marsden, T. (2004). Exploring the "limits to growth" in UK organics: beyond the statistical image. Journal of Rural Studies, 20(3), 345-357.

SSPA (2017). Combatir con éxito la despoblación mediante un nuevo modelo de desarrollo territorial. La experiencia de Highlands and Islands Enterprise. Informe del grupo de visita TAIEX-REGIO de Cuenca, Euritania, Lika-Senj, Soria y Teruel a las Tierras Altas e Islas de Escocia, 2-4 de mayo de 2017. Red de Áreas Escasamente Pobladas del Sur de Europa (Southern Sparsely Populated Areas -SSPA-). Disponible en: http://sspanetwork.eu/wp-content/uploads/HIE-SSPA-Informe-completo.pdf

TEAGASC (2011). About. Disponible en: https://www.teagasc.ie/about/

Tolón, A. y Lastra, X. (2007). Evolución del desarrollo rural en Europa y en España. Las áreas rurales de la metodología LEADER. Revista Electrónica de Medio Ambiente UCM, 4, 3562.

Tribunal de Cuentas Europeo (2018). Energía renovable para un desarrollo rural sostenible: posibles sinergias significativas, pero en su mayoría no materializadas en la práctica, Informe especial 05/2018. Luxembourg: Publications Office of the European Union. Disponible

en: https://www.eca.europa.eu/Lists/ECADocuments/SR18_05/SR_Renewable_Energy_ ES.pdf

Van der ploeg, J. D., Renting, H., Brunori, G., Knickel, K., Mannion, J., Marsden, T., Roest, K., de Sevilla-Guzmán, E., Ventura, F. (2000). Rural development: from practices and policies towards theory. Sociologia Ruralis, 40, 391-408.

Van der sangen, G. J. H. (2013). The Netherlands en International Handbook of Cooperative Law. Berlin/Heidelberg, Springer, pp. 541-561.

Volkman, K. (2001). Tourism in Ireland: Observations on the Impact of European Union. Funding and Marketing Strategies. ASAC. Londres. 
Wanhill S. (1977). Peripheral area tourism: a European perspective. Progress in Tourism and Hospitality Research, 3, 47-70.

Zanoli, R. (2004). The European consumer and organic food. Organic Marketing Iniciatives and Rural Development (OMIaRD), 4, University of Wales Aberystwyth. 